\title{
STABILIZATION OF NETWORKED CONTROL SYSTEMS VIA DYNAMIC OUTPUT-FEEDBACK CONTROLLERS*
}

\author{
HUIJUN $\mathrm{GAO}^{\dagger}$, XIANGYU MENG ${ }^{\ddagger}$, TONGWEN CHEN ${ }^{\S}$, AND JAMES LAM
}

\begin{abstract}
This paper investigates the problem of stabilization of networked control systems via dynamic output-feedback controllers. The physical plant and the dynamic controller are in continuous time, and a communication channel exists between the output of the physical plant and the input of the dynamic controller. Three important communication features are considered: measurement quantization, signal transmission delay, and data packet dropout, which appear typically in a networked environment. Attention is focused on the design of dynamic output-feedback controllers which ensure asymptotic stability of the closed-loop systems. Linear matrix inequality (LMI)-based conditions are formulated for the existence of admissible controllers. If these conditions are satisfied, a desired controller can be readily constructed. A satellite system is used to illustrate the applicability and effectiveness of the proposed controller design method.
\end{abstract}

Key words. data packet dropout, dynamic output-feedback control, networked control systems, quantization, signal transmission delay

AMS subject classifications. 93D15, 93D05, 93D20

DOI. $10.1137 / 070679132$

1. Introduction. One common assumption in traditional control systems is that the interconnection between plants and controllers is transparent, based on which transmitted signals are equal to received signals. This assumption has been used implicitly for a few decades and has underlain many successful control designs for either linear or nonlinear dynamic systems. In some situations, however, transparent communication is not always guaranteed, and a communication link sometimes constitutes a bottleneck in control system operations. Therefore, networked control systems (NCSs) appeared recently and have been drawing more and more attention from researchers working in the areas of systems and control, highlighted by the recent special issue [1] and survey papers [23, 29].

When a communication link is involved in control system design, usually three important aspects need to be taken into account. The first one is signal transmission delay, which is usually caused by the limited bit rate of the communication channel, by a node waiting to send out a packet via a busy channel, or by signal processing and propagation. The second interesting problem in a communication channel is the data packet dropout (or data missing $[25,26]$ ) phenomenon, which is usually caused

*Received by the editors January 4, 2007; accepted for publication (in revised form) November 10, 2009; published electronically March 5, 2010. This work was supported by Natural Sciences and Engineering Research Council of Canada, National Natural Science Foundation of China (60825303, 60834003), National Basic Research Program of China (2009CB320600), the 111 Project (B08015), China Scholarship Council, and Research Grants Council under grant HKU 7031/07P.

http://www.siam.org/journals/sicon/48-5/67913.html

${ }^{\dagger}$ Department of Control Science and Engineering, Harbin Institute of Technology, Harbin, Heilongjiang, 150001, China (hjgao@hit.edu.cn).

${ }_{\ddagger}^{\ddagger}$ Department of Control Science and Engineering, Harbin Institute of Technology, Harbin, Heilongjiang, 150001, China and Department of Electrical and Computer Engineering, University of Alberta, Edmonton, Alberta, T6G 2V4, Canada (xmeng2@ece.ualberta.ca)

$\S$ Department of Electrical and Computer Engineering, University of Alberta, Edmonton, Alberta, T6G 2V4, Canada (tchen@ece.ualberta.ca).

I Department of Mechanical Engineering, University of Hong Kong, Hong Kong (james.lam@hku. hk). 
by the unavoidable errors or losses in the transmission. Though many networked systems employ automatic repeat request mechanisms, packet dropout phenomenon is still unavoidable. Moreover, packets may deliberately be dropped if a packet sampled at the sensor node reaches the destination later than its successors, since it is natural to use the most updated packet by dropping out the old ones. Another important issue in a communication channel is the quantization effect. In a networked environment, signals are usually quantized before being communicated, and the number of quantization levels is closely related to the information flow between the components of the system and thus to the capacity required to transmit the information. In summary, signal transmission delay, data packet dropout, and signal quantization are three important issues to be addressed for communication based systems.

For NCSs, a number of important pieces of work have been reported [3, 12, 15, 16, $17,18,19,21,22,24,28,30,31,32,33,34]$. Earlier work mainly focused on stability analysis and performance [9], while more recently, attention has been devoted to controller synthesis. These pieces of work have significant importance on both theoretical advancement and practical applications of NCSs. It is worth noting, however, that the results obtained for NCSs are still very limited: (i) most of the aforementioned results consider only one or two aspects of the communication issues, while few papers address the control problems with the simultaneous consideration of the three important communication features mentioned above; (ii) most of the obtained results are concerned with the problem of state-feedback control [8], which is only applicable for systems whose state variables can be measured online, while few papers tackle the problem of output-feedback control, which is more useful and challenging than the state-feedback case. Motivated by the above observations, it is our intention to solve the problem of dynamic output-feedback stabilization for NCSs with the simultaneous presence of signal transmission delay, data packet dropout, and measurement quantization. We characterize physical systems in a deterministic manner, but another important and well-developed field of networked controls is stochastic networked control systems. Key references describing the stochastic networked systems include [4, 20, 22].

In this paper, we investigate the problem of stabilization of networked control systems via dynamic output-feedback centralized controllers. In this configuration, there is no local controller for each plant, and the centralized controller makes decisions based on sensor information which is sent over the network. The physical plant and the dynamic controller are in continuous time, and a communication channel is present in the loop between the output of the physical plant and the input of the dynamic controller. All three important communication features mentioned above are taken into consideration and tackled in a unified framework. Attention is focused on the design of dynamic output-feedback controllers which ensure asymptotic stability of the closed-loop systems; linear matrix inequality (LMI)-based conditions are formulated for the existence of admissible controllers. If these conditions are feasible, a desired controller can be readily constructed. Finally, a satellite system is used to illustrate the applicability and effectiveness of the proposed controller design.

Note. The notation used throughout the paper is standard. The superscripts " $T$ " and " -1 " stand for matrix transposition and matrix inverse, respectively, $\mathbb{R}^{n}$ denotes the $n$-dimensional Euclidean space, and the notation $P>0(\geq 0)$ means that $P$ is real symmetric and positive definite (semidefinite). The symmetric term in a symmetric matrix is denoted by *, e.g.,

$$
\left[\begin{array}{ll}
X & Y \\
* & Z
\end{array}\right]=\left[\begin{array}{cc}
X & Y \\
Y^{T} & Z
\end{array}\right]
$$




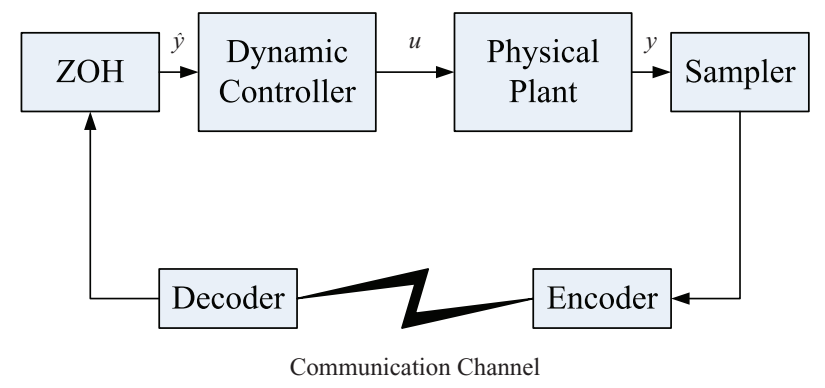

FIG. 1. Problem of networked output-feedback stabilization.

and $\operatorname{diag}\{\ldots\}$ stands for a block-diagonal matrix. In addition, $I$ denotes an identity matrix. Matrices, if their dimensions are not explicitly stated, are assumed to be compatible for algebraic operations.

2. Problem formulation. The problem of network-based output-feedback stabilization is shown in Figure 1. In this figure, $u$ and $y$ denote the control input and measured output of the physical plant, respectively; $\hat{y}$ denotes the input of the dynamic controller. The measured output $y$ is sampled periodically and then transmitted through a network medium to the $\mathrm{ZOH}$ (zero-order hold), whose output $\hat{y}$ is used as the input of the dynamic controller. We consider a communication channel with the occurrence of packet dropouts and delays where they may vary within the bounds on the number of consecutive packet dropouts and on the size of each delay. In our problem, we use a quantizer to characterize the coding effect, which is induced by the encoder and decoder used in the whole process. We consider logarithmic quantizer and zero-order hold, and we assume the behavior of sample and $\mathrm{ZOH}$ to be deterministic. This configuration with single-side network connection has possible applications in wireless networked control systems.

Suppose the physical plant is given by

$$
\begin{aligned}
\mathcal{S}: \quad \dot{x}(t) & =A x(t)+B u(t), \\
y(t) & =C x(t) .
\end{aligned}
$$

Here $x(t) \in \mathbb{R}^{n}$ is the state vector; $y(t) \in \mathbb{R}^{m}$ is the measured output; $u(t) \in \mathbb{R}^{p}$ is the control input; $A, B, C$ are system matrices with appropriate dimensions.

From Figure 1, we see that there is a communication channel between $y(t)$ and $\hat{y}(t)$. As discussed above, generally, three effects need to be taken into consideration: signal quantization, signal transmission delay, and data packet dropout. It is assumed that the sampler is clock-driven, while the $\mathrm{ZOH}$ is event-driven. The sampling period for $y(t)$ is assumed to be $h$, with $h$ being a positive constant. Denote the updating instants of the $\mathrm{ZOH}$ as $t_{k}, k=1, \ldots, \infty$, and suppose that the updating signal (successfully transmitted signal from the sampler to the $\mathrm{ZOH}$ ) at the instant $t_{k}$ has experienced signal transmission delay $\beta_{k}$. A natural assumption on the signal transmission delay $\beta_{k}$ can be made as follows:

$$
0 \leq \beta_{k} \leq \bar{\beta},
$$

where $\bar{\beta}$ denotes the upper delay bound.

In what follows, we model signal quantization, signal transmission delay, and data packet dropout mathematically. 
2.1. Signal quantization. It is assumed that the sampled measurements of $y(t)$ are firstly encoded using a quantizer (the quantizer constructed on each channel), which is denoted as $f(\cdot)=\left[\begin{array}{llll}f_{1}(\cdot) & f_{2}(\cdot) & \ldots & f_{m}(\cdot)\end{array}\right]^{T}$ and then transmitted with a single packet. The quantization effect of the encoding and decoding process is assumed to be time-invariant, logarithmic, and symmetric; i.e., $f(-v)=-f(v)$. For each $f_{j}(\cdot)$, given constants $u_{0}^{(j)}$ and $\rho_{j}$ such that $u_{0}^{(j)}>0$ and $0<\rho_{j}<1$, the set of quantized levels is characterized by $[5,6]$

$$
\mathcal{U}_{j}=\left\{ \pm u_{i}^{(j)}, \quad u_{i}^{(j)}=\rho_{j}^{i} u_{0}^{(j)}, \quad i= \pm 1, \pm 2, \ldots\right\} \cup\left\{ \pm u_{0}^{(j)}\right\} \cup\{0\}
$$

It is noted that $u_{-i}^{(j)}=\rho_{j}^{-i} u_{0}^{(j)} \neq-u_{i}^{(j)}$. Given the set of quantized levels, we define the associated logarithmic quantizer $f_{j}(\cdot): \mathbb{R} \rightarrow \mathcal{U}_{j}$ as follows:

$$
f_{j}(v)= \begin{cases}u_{i}^{(j)} & \text { if } \frac{1}{1+\sigma_{j}} u_{i}^{(j)}<v \leq \frac{1}{1-\sigma_{j}} u_{i}^{(j)}, \quad v>0, \quad i \in \mathbb{Z} \\ 0 & \text { if } v=0 \\ -f_{j}(-v) & \text { if } v<0\end{cases}
$$

where

$$
\sigma_{j}=\frac{1-\rho_{j}}{1+\rho_{j}}
$$

Each of the quantization level $u_{i}^{(j)}$ corresponds to a interval $\left(\frac{1+\rho_{j}}{2} u_{i}^{(j)}, \frac{1+\rho_{j}}{2} \frac{1}{\rho_{j}} u_{i}^{(j)}\right]$ such that the quantizer maps the whole interval to this quantization level. In addition, these intervals

$$
\left(\cup_{i \in \mathbb{Z}}\left[-\frac{1+\rho_{j}}{2} \rho_{j}^{i-1} u_{0}^{(j)},-\frac{1+\rho_{j}}{2} \rho_{j}^{i} u_{0}^{(j)}\right)\right) \cup 0 \cup\left(\cup_{i \in \mathbb{Z}}\left(\frac{1+\rho_{j}}{2} \rho_{j}^{i} u_{0}^{(j)}, \frac{1+\rho_{j}}{2} \rho_{j}^{i-1} u_{0}^{(j)}\right]\right)
$$

form a partition of $\mathbb{R}$; that is, they are disjoint, and their union for $i$ equals to $\mathbb{R}$. Figure 2 gives a visual sketch clarifying this statement.

2.2. Signal transmission delay. At the updating instant $t_{k}$, we have the following relationship:

$$
\hat{y}\left(t_{k}\right)=f\left(y\left(t_{k}-\beta_{k}\right)\right) .
$$

Thus, considering the behavior of the $\mathrm{ZOH}$, we have

$$
\hat{y}(t)=f\left(y\left(t_{k}-\beta_{k}\right)\right), \quad t_{k} \leq t<t_{k+1},
$$

with $t_{k+1}$ being the next updating instant of the $\mathrm{ZOH}$ after $t_{k}$.

2.3. Data packet dropout. At the updating instant $t_{k}$, the number of accumulated data packet dropouts since the last updating instant $t_{k-1}$ is denoted as $\delta_{k}$. We assume that the maximum number of consecutive data packet dropouts is $\bar{\delta}$, that is,

$$
0 \leq \delta_{k} \leq \bar{\delta}
$$

Then, it can be seen from (2) and (8) that

$$
h \leq t_{k+1}-t_{k} \leq \bar{\beta}+(\bar{\delta}+1) h,
$$




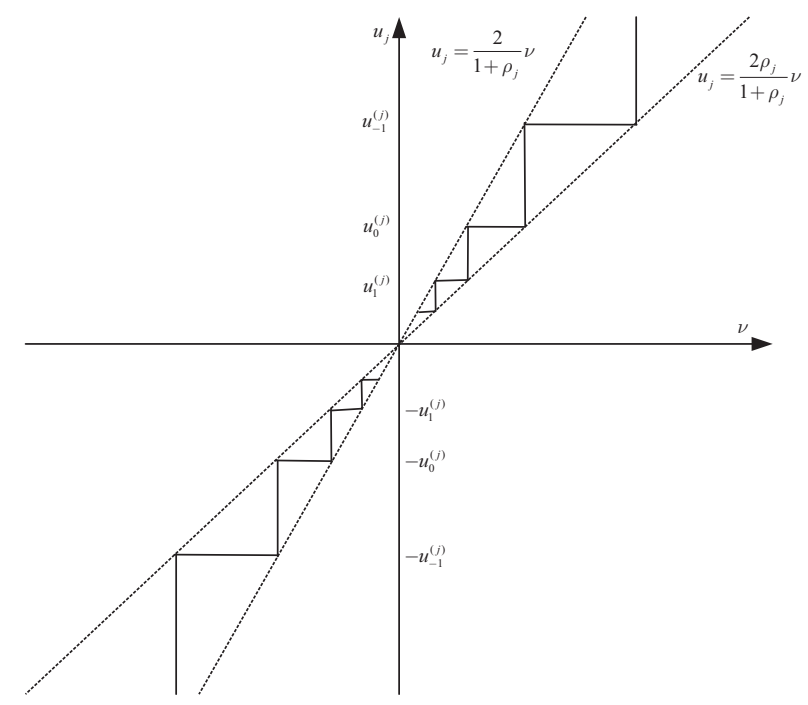

FIG. 2. Logarithmic quantizer.

which implies that the interval between any two successive updating instants is upper bounded by $\bar{\beta}+(\bar{\delta}+1) h$ and lower bounded by $h$.

In this paper, it is assumed that not all the state variables of the physical plant can be measured online, and we consider the following dynamic output-feedback controller:

$$
\begin{aligned}
\mathcal{C}: \quad \dot{x}_{c}(t) & =A_{c} x_{c}(t)+A_{d} x_{c}\left(t_{k}-\beta_{k}\right)+B_{c} \hat{y}(t), \\
u(t) & =C_{c} x_{c}(t), \quad t_{k} \leq t<t_{k+1},
\end{aligned}
$$

where $x_{c}(t) \in \mathbb{R}^{n}$ is the state vector of the dynamic controller; $A_{c}, A_{d}, B_{c}$, and $C_{c}$ are appropriately dimensioned controller matrices to be determined. It is worth mentioning that in our approach, the introduction of the term $A_{d} x_{c}\left(t_{k}-\beta_{k}\right)$ is essential to make the controller synthesis tractable. By adding a time stamp to the data at the sampler, we can trace the time instant $t_{k}-\beta_{k}$, which means that the controller in (10) is online implementable. We assume that the time stamp associated with the values is not quantized. This assumption simplifies the problem and allows us to convert the quantized feedback design problem to a robust control problem with sector bound uncertainties. The more realistic case, where the time is quantized as well, is still a challenging problem to be investigated.

Then, the problem to be addressed in this paper can be expressed as follows.

Problem networked output-feedback stabilization. Consider the problem of network-based output-feedback stabilization in Figure 1 and system $\mathcal{S}$ in (1). The sampler, encoder, decoder, and $\mathrm{ZOH}$ are configured in the networked control system, and all the parameters $\left(h, \bar{\beta}, u_{0}^{(j)}, \rho_{j}\right.$, and $\left.\bar{\delta}\right)$ are given. The objective is to determine the matrices $A_{c}, A_{d}, B_{c}, C_{c}$ of the controller $\mathcal{C}$ in (10) such that the closed-loop system is asymptotically stable for all admissible signal quantizations, signal transmission delays, and data packet dropouts.

Before proceeding further, we give the following lemma which will be used later $[10,27]$. 
LEMMA 2.1. Given appropriately dimensioned matrices $\Sigma_{1}, \Sigma_{2}, \Sigma_{3}$, with $\Sigma_{1}^{T}=$ $\Sigma_{1}$, then,

$$
\Sigma_{1}+\Sigma_{3} F(t) \Sigma_{2}+\Sigma_{2}^{T} F^{T}(t) \Sigma_{3}^{T}<0
$$

holds for all $F(t)$ satisfying $F^{T}(t) F(t) \leq I$ if and only if for some $\epsilon>0$,

$$
\Sigma_{1}+\epsilon^{-1} \Sigma_{3} \Sigma_{3}^{T}+\epsilon \Sigma_{2}^{T} \Sigma_{2}<0
$$

3. Main results. We are first concerned with the analysis problem. More specifically, assuming that the controller matrices $\left(A_{c}, A_{d}, B_{c}, C_{c}\right)$ are known, we shall study the conditions under which the closed-loop system is asymptotically stable for all admissible measurement quantizations, signal transmission delays, and data packet dropouts. The following lemma shows that the asymptotic stability of the closed-loop system can be guaranteed if there exist some matrix variables satisfying certain LMIs. This lemma will play an instrumental role in the controller synthesis.

LEMMA 3.1. Consider the problem of network-based output-feedback stabilization in Figure 1. Given the parameters $h, \bar{\beta}, u_{0}^{(j)}, \rho_{j}, \bar{\delta}$ and controller matrices $A_{c}, A_{d}$, $B_{c}, C_{c}$, the closed-loop system is asymptotically stable if there exist matrices $P>0$, $Z>0, U, W$, and a scalar $\epsilon>0$ satisfying

$$
\left[\begin{array}{ccccc}
\operatorname{sym}(P \bar{A}+U) & P \bar{B}-U+W^{T} & \sqrt{\tilde{\beta}} U & \sqrt{\tilde{\beta}} \bar{A}^{T} Z & P B_{\Lambda} \\
* & -\operatorname{sym}(W)+\epsilon C_{\Lambda}^{T} \Lambda^{2} C_{\Lambda} & \sqrt{\tilde{\beta}} W & \sqrt{\tilde{\beta}} \bar{B}^{T} Z & 0 \\
* & * & -Z & 0 & 0 \\
* & * & * & -Z & \sqrt{\tilde{\beta}} Z B_{\Lambda} \\
* & * & * & * & -\epsilon I
\end{array}\right]<0
$$

where

$$
\begin{aligned}
& \bar{A}=\left[\begin{array}{cc}
A & B C_{c} \\
0 & A_{c}
\end{array}\right], \quad \bar{B}=\left[\begin{array}{cc}
0 & 0 \\
B_{c} C & A_{d}
\end{array}\right], \quad B_{\Lambda}=\left[\begin{array}{c}
0 \\
B_{c}
\end{array}\right], \quad C_{\Lambda}=\left[\begin{array}{ll}
C & 0
\end{array}\right], \\
& \Lambda=\operatorname{diag}\left\{\sigma_{1}, \ldots, \sigma_{m}\right\}, \quad \tilde{\beta}=2 \bar{\beta}+(\bar{\delta}+1) h, \quad \text { and } \quad \operatorname{sym}(X)=X+X^{T} .
\end{aligned}
$$

Proof. First, by substituting (7) into (10), we obtain

$$
\begin{aligned}
\dot{x}_{c}(t) & =A_{c} x_{c}(t)+A_{d} x_{c}\left(t_{k}-\beta_{k}\right)+B_{c} f\left(y\left(t_{k}-\beta_{k}\right)\right), \\
u(t) & =C_{c} x_{c}(t), \quad t_{k} \leq t<t_{k+1} .
\end{aligned}
$$

Considering the quantization behavior shown in (3)-(5) and according to [5, 6], (14) can be expressed as

$$
\begin{aligned}
\dot{x}_{c}(t) & =A_{c} x_{c}(t)+A_{d} x_{c}\left(t_{k}-\beta_{k}\right)+B_{c}(I+\Lambda(t)) y\left(t_{k}-\beta_{k}\right), \\
u(t) & =C_{c} x_{c}(t), \quad t_{k} \leq t<t_{k+1}
\end{aligned}
$$

where

$$
\begin{aligned}
\Lambda(t) & =\operatorname{diag}\left\{\Lambda_{1}(t), \quad \Lambda_{2}(t), \ldots, \Lambda_{m}(t)\right\} \\
\Lambda_{j}(t) & \in\left[-\sigma_{j}, \sigma_{j}\right], \quad j=1, \ldots, m .
\end{aligned}
$$

Copyright (C) by SIAM. Unauthorized reproduction of this article is prohibited. 
Let us represent $t_{k}-\beta_{k}$ in (15) as

$$
t_{k}-\beta_{k}=t-\beta(t)
$$

where

$$
\beta(t)=t-t_{k}+\beta_{k}
$$

Then, from (2), (8), (9), and (13) we have

$$
0 \leq \beta(t) \leq \tilde{\beta} .
$$

By substituting (17) into (15), we obtain

$$
\begin{aligned}
\dot{x}_{c}(t) & =A_{c} x_{c}(t)+A_{d} x_{c}(t-\beta(t))+B_{c}(I+\Lambda(t)) y(t-\beta(t)), \\
u(t) & =C_{c} x_{c}(t) .
\end{aligned}
$$

By connecting (18) and (1), the closed-loop system can be written as

$$
\dot{\xi}(t)=\bar{A} \xi(t)+\left(\bar{B}+B_{\Lambda} \Lambda(t) C_{\Lambda}\right) \xi(t-\beta(t)),
$$

where $\xi(t)=\left[\begin{array}{ll}x^{T}(t) & x_{c}^{T}(t)\end{array}\right]^{T}$ and $\bar{A}, \bar{B}, B_{\Lambda}, C_{\Lambda}$ are given in (13).

Now, choose the following Lyapunov-Krasovskii functional [13, 14]:

$$
V(t)=\xi^{T}(t) P \xi(t)+\int_{-\tilde{\beta}}^{0} \int_{t+\omega}^{t} \dot{\xi}^{T}(\lambda) Z \dot{\xi}(\lambda) d \lambda d \omega,
$$

where $P>0, Z>0$ are matrices to be determined. Then, along the solution of the closed-loop system in (19), the time derivative of $V(t)$ is given by

$$
\begin{aligned}
\dot{V}(t) & =2 \xi^{T}(t) P \dot{\xi}(t)+\tilde{\beta} \dot{\xi}^{T}(t) Z \dot{\xi}(t)-\int_{t-\tilde{\beta}}^{t} \dot{\xi}^{T}(\lambda) Z \dot{\xi}(\lambda) d \lambda \\
& \leq 2 \xi^{T}(t) P \dot{\xi}(t)+\tilde{\beta} \dot{\xi}^{T}(t) Z \dot{\xi}(t)-\int_{t-\beta(t)}^{t} \dot{\xi}^{T}(\lambda) Z \dot{\xi}(\lambda) d \lambda .
\end{aligned}
$$

By the Newton-Leibniz formula, we have

$$
\int_{t-\beta(t)}^{t} \dot{\xi}(\lambda) d \lambda=\xi(t)-\xi(t-\beta(t)) .
$$

Then, for any appropriately dimensioned matrices $M=\left[\begin{array}{c}U \\ W\end{array}\right]$, we have

$$
2 \phi^{T}(t) M\left[\xi(t)-\xi(t-\beta(t))-\int_{t-\beta(t)}^{t} \dot{\xi}(\lambda) d \lambda\right]=0,
$$

where $\phi(t)=\left[\begin{array}{ll}\xi^{T}(t) & \xi^{T}(t-\beta(t))\end{array}\right]^{T}$. Then, from (19), (21), and (22) we obtain

$$
\begin{aligned}
\dot{V}(t) \leq & 2 \xi^{T}(t) P \dot{\xi}(t)+\tilde{\beta} \dot{\xi}^{T}(t) Z \dot{\xi}(t)-\int_{t-\beta(t)}^{t} \dot{\xi}^{T}(\lambda) Z \dot{\xi}(\lambda) d \lambda \\
& +2 \phi^{T}(t) M\left[\xi(t)-\xi(t-\beta(t))-\int_{t-\beta(t)}^{t} \dot{\xi}(\lambda) d \lambda\right] \\
\leq & \phi^{T}(t)\left(\Gamma+\tilde{\beta} M Z^{-1} M^{T}\right) \phi(t) \\
& -\int_{t-\beta(t)}^{t}\left[\phi^{T}(t) M+\dot{\xi}^{T}(\lambda) Z\right] Z^{-1}\left[M^{T} \phi(t)+Z \dot{\xi}(\lambda)\right] d \lambda,
\end{aligned}
$$

Copyright $@$ ㅇ by SIAM. Unauthorized reproduction of this article is prohibited. 
where

$$
\begin{aligned}
\Gamma & =\Gamma_{1}+\Gamma_{2}+\Gamma_{2}^{T}+\tilde{\beta} \Gamma_{3}^{T} Z \Gamma_{3}, \quad \Gamma_{1}=\left[\begin{array}{cc}
P \bar{A}+\bar{A}^{T} P & P\left(\bar{B}+B_{\Lambda} \Lambda(t) C_{\Lambda}\right) \\
* & 0
\end{array}\right], \\
\Gamma_{2} & =\left[\begin{array}{ll}
M & -M
\end{array}\right], \quad \Gamma_{3}=\left[\begin{array}{lll}
\bar{A} & \bar{B}+B_{\Lambda} \Lambda(t) C_{\Lambda}
\end{array}\right] .
\end{aligned}
$$

By noticing that $Z>0$, we have $\left[\phi^{T}(t) M+\dot{\xi}^{T}(\lambda) Z\right] Z^{-1}\left[M^{T} \phi(t)+Z \dot{\xi}(\lambda)\right] \geq 0$. Therefore, from (23) we know that $\dot{V}(t)<0$ if

$$
\Gamma+\tilde{\beta} M Z^{-1} M^{T}<0
$$

which, by the Schur complement, is equivalent to

$$
\left[\begin{array}{ccc}
\Gamma_{1}+\Gamma_{2}+\Gamma_{2}^{T} & \sqrt{\tilde{\beta}} M & \sqrt{\tilde{\beta}} \Gamma_{3}^{T} Z \\
* & -Z & 0 \\
* & * & -Z
\end{array}\right]<0 .
$$

Now, rewrite (24) in the form of (11) with

$$
\begin{aligned}
\Sigma_{1} & =\left[\begin{array}{cccc}
P \bar{A}+\bar{A}^{T} P+U+U^{T} & P \bar{B}-U+W^{T} & \sqrt{\tilde{\beta}} U & \sqrt{\tilde{\beta}} \bar{A}^{T} Z \\
* & -W-W^{T} & \sqrt{\tilde{\beta}} W & \sqrt{\tilde{\beta}} \bar{B}^{T} Z \\
* & * & -Z & 0 \\
* & * & * & -Z
\end{array}\right], \\
\Sigma_{3} & =\left[\begin{array}{c}
P B_{\Lambda} \\
0 \\
0 \\
\sqrt{\tilde{\beta}} Z B_{\Lambda}
\end{array}\right], \quad \Sigma_{2}=\left[\begin{array}{llll}
0 & \Lambda C_{\Lambda} & 0 & 0
\end{array}\right], \quad F(t)=\Lambda(t) \Lambda^{-1} .
\end{aligned}
$$

By Lemma 2.1 together with a Schur complement operation, (24) holds if for some $\epsilon>0,(12)$ holds, and the proof is completed.

It is noted that if the controller matrices $\left(A_{c}, A_{d}, B_{c}, C_{c}\right)$ are given, the conditions in Lemma 3.1 are LMIs over the decision variables $P>0, Z \geq 0, U, W$, and scalar $\epsilon>0$. However, since our eventual purpose is to determine the controller matrices $\left(A_{c}, A_{d}, B_{c}, C_{c}\right)$, the above conditions are actually nonlinear matrix inequalities. Our main objective hereafter is to transform them into tractable conditions to solve the control synthesis problem.

THEOREM 3.2. Consider the problem of network-based output-feedback stabilization in Figure 1 . Given the parameters $h, \bar{\beta}, u_{0}^{(j)}, \rho_{j}$, and $\bar{\delta}$, a dynamic controller in the form of (10) exists such that the closed-loop system is asymptotically stable if there exist matrices $X>0, Y>0, \bar{Z}=\left[\begin{array}{cc}Z_{1} & Z_{2} \\ * & Z_{3}\end{array}\right]>0, \bar{U}=\left[\begin{array}{cc}U_{1} & U_{2} \\ U_{4} & U_{3}\end{array}\right], \bar{W}=\left[\begin{array}{cc}W_{1} & W_{2} \\ W_{4} & W_{3}\end{array}\right]$, $\bar{A}_{c}, \bar{A}_{d}, \bar{B}_{c}, \bar{C}_{c}$, and a scalar $\epsilon>0$ satisfying

$$
\left.\begin{array}{cccccc}
\Psi_{11} & \Psi_{12} & \sqrt{\tilde{\beta}} \bar{U} & \Psi_{14} & \Psi_{15} & 0 \\
* & \Psi_{22} & \sqrt{\tilde{\beta}} \bar{W} & \Psi_{24} & 0 & \Psi_{26} \\
* & * & -\bar{Z} & 0 & 0 & 0 \\
* & * & * & \Psi_{44} & \Psi_{45} & 0 \\
* & * & * & * & -\epsilon I & 0 \\
* & * & * & * & * & -\epsilon^{-1} I
\end{array}\right]<0,
$$

Copyright $@$ by SIAM. Unauthorized reproduction of this article is prohibited. 
where

$$
\begin{aligned}
& \Psi_{11}=\left[\begin{array}{cc}
A X+B \bar{C}_{c}+X A^{T}+\bar{C}_{c}^{T} B^{T}+U_{1}+U_{1}^{T} & A+\bar{A}_{c}^{T}+U_{2}+U_{4}^{T} \\
* & Y A+A^{T} Y+U_{3}+U_{3}^{T}
\end{array}\right], \\
& \Psi_{12}=\left[\begin{array}{cc}
-U_{1}+W_{1}^{T} & -U_{2}+W_{4}^{T} \\
\bar{A}_{d}-U_{4}+W_{2}^{T} & \bar{B}_{c} C-U_{3}+W_{3}^{T}
\end{array}\right], \quad \Psi_{15}=\left[\begin{array}{c}
0 \\
\bar{B}_{c}
\end{array}\right], \\
& \Psi_{22}=\left[\begin{array}{cc}
-W_{1}-W_{1}^{T} & -W_{2}-W_{4}^{T} \\
* & -W_{3}-W_{3}^{T}
\end{array}\right], \quad \Psi_{26}=\left[\begin{array}{c}
X C^{T} \Lambda \\
C^{T} \Lambda
\end{array}\right], \\
& \Psi_{14}=\left[\begin{array}{cc}
\sqrt{\tilde{\beta}} X A^{T}+\sqrt{\tilde{\beta}} \bar{C}_{c}^{T} B^{T} & \sqrt{\tilde{\beta}} \bar{A}_{c}^{T} \\
\sqrt{\tilde{\beta}} A^{T} & \sqrt{\tilde{\beta}} A^{T} Y
\end{array}\right], \quad \Psi_{24}=\left[\begin{array}{cc}
0 & \sqrt{\tilde{\beta}} \bar{A}_{d}^{T} \\
0 & \sqrt{\tilde{\beta}} C^{T} \bar{B}_{c}^{T}
\end{array}\right], \\
& \Psi_{44}=\left[\begin{array}{cc}
Z_{1}-2 X & Z_{2}-2 I \\
* & Z_{3}-2 Y
\end{array}\right], \quad \Psi_{45}=\left[\begin{array}{c}
0 \\
\sqrt{\tilde{\beta}} \bar{B}_{c}
\end{array}\right],
\end{aligned}
$$

and $\Lambda$ and $\tilde{\beta}$ are given in (13). Moreover, if the above conditions are satisfied, a desired output-feedback controller is given in the form of (10), with parameters as follows:

$$
\begin{aligned}
& A_{c}=S^{-1}\left(\bar{A}_{c}-Y A X-Y B \bar{C}_{c}\right) U^{-T}, \quad B_{c}=S^{-1} \bar{B}_{c}, \\
& A_{d}=S^{-1}\left(\bar{A}_{d}-\bar{B}_{c} C X\right) U^{-T}, \quad C_{c}=\bar{C}_{c} U^{-T}
\end{aligned}
$$

where $S$ and $U$ are any nonsingular matrices satisfying

$$
S U^{T}=I-Y X .
$$

Proof. Suppose that conditions (25) and (26) are satisfied; we will prove that the controller in the form of (10) with parameters given in (28) guarantees the closed-loop system to be asymptotically stable. By the Schur complement, (26) implies $Y-X^{-1}>$ 0 , and thus $I-Y X$ is nonsingular. Therefore, there always exist nonsingular matrices $S$ and $U$ satisfying (29). Now introduce the following nonsingular matrices:

$$
\Pi_{1}=\left[\begin{array}{cc}
X & I \\
U^{T} & 0
\end{array}\right], \quad \Pi_{2}=\left[\begin{array}{cc}
I & Y \\
0 & S^{T}
\end{array}\right] .
$$

Let

$$
P=\Pi_{2} \Pi_{1}^{-1}
$$

Then, it can be verified that

$$
P=\left[\begin{array}{cc}
Y & S \\
S^{T} & \Upsilon
\end{array}\right]
$$

where $\Upsilon=U^{-1} X\left(Y-X^{-1}\right) X U^{-T}>0$. In addition, we have

$$
\Upsilon-S^{T} Y^{-1} S=S^{T}(I-X Y)^{-1}\left(X-Y^{-1}\right)(I-Y X)^{-1} S>0,
$$

which implies by the Schur complement that $P>0$. From (28), we have

$$
\begin{aligned}
& \bar{A}_{c}=Y A X+Y B C_{c} U^{T}+S A_{c} U^{T}, \\
& \bar{A}_{d}=S B_{c} C X+S A_{d} U^{T}, \quad \bar{B}_{c}=S B_{c}, \quad \bar{C}_{c}=C_{c} U^{T} .
\end{aligned}
$$

Copyright $@$ by SIAM. Unauthorized reproduction of this article is prohibited. 
Define

$$
Z=\Pi_{1}^{-T} \bar{Z} \Pi_{1}^{-1}, \quad U=\Pi_{1}^{-T} \bar{U} \Pi_{1}^{-1}, \quad W=\Pi_{1}^{-T} \bar{W} \Pi_{1}^{-1} .
$$

Substituting the above matrices $\bar{A}_{c}, \bar{A}_{d}, \bar{B}_{c}, \bar{C}_{c}$ into (25), and by calculation, we have that $(25)$ is equivalent to

$$
\left[\begin{array}{cccccc}
\Sigma_{11} & \Sigma_{12} & \sqrt{\tilde{\beta}} \Pi_{1}^{T} U \Pi_{1} & \sqrt{\tilde{\beta}} \Pi_{1}^{T} \bar{A}^{T} \Pi_{2} & \Pi_{2}^{T} B_{\Lambda} & 0 \\
* & \Sigma_{22} & \sqrt{\tilde{\beta}} \Pi_{1}^{T} W \Pi_{1} & \sqrt{\tilde{\beta}} \Pi_{1}^{T} \bar{B}^{T} \Pi_{2} & 0 & \Pi_{1}^{T} C_{\Lambda}^{T} \Lambda \\
* & * & -\Pi_{1}^{T} Z \Pi_{1} & 0 & 0 & 0 \\
* & * & * & \Pi_{1}^{T} Z \Pi_{1}-2 \Pi_{1}^{T} \Pi_{2} & \sqrt{\tilde{\beta}} \Pi_{2}^{T} B_{\Lambda} & 0 \\
* & * & * & * & -\epsilon I & 0 \\
* & * & * & * & * & -\epsilon^{-1} I
\end{array}\right]<0
$$

where

$$
\begin{aligned}
\Sigma_{11} & =\Pi_{2}^{T} \bar{A} \Pi_{1}+\Pi_{1}^{T} \bar{A}^{T} \Pi_{2}+\Pi_{1}^{T} U \Pi_{1}+\Pi_{1}^{T} U^{T} \Pi_{1}, \\
\Sigma_{12} & =\Pi_{2}^{T} \bar{B} \Pi_{1}-\Pi_{1}^{T} U \Pi_{1}+\Pi_{1}^{T} W^{T} \Pi_{1}, \\
\Sigma_{22} & =-\Pi_{1}^{T} W \Pi_{1}-\Pi_{1}^{T} W^{T} \Pi_{1} .
\end{aligned}
$$

Performing a congruence transformation to (31) by $\operatorname{diag}\left\{\Pi_{1}^{-1}, \Pi_{1}^{-1}, \Pi_{1}^{-1}, \Pi_{1}^{-1}, I, I\right\}$ with the consideration of (30) and by a Schur complement operation, we obtain

(32)

$$
\left[\begin{array}{ccccc}
\operatorname{sym}(P \bar{A}+U) & P \bar{B}-U+W^{T} & \sqrt{\tilde{\beta}} U & \sqrt{\tilde{\beta}} \bar{A}^{T} P & P B_{\Lambda} \\
* & -\operatorname{sym}(W)+\epsilon C_{\Lambda}^{T} \Lambda^{2} C_{\Lambda} & \sqrt{\tilde{\beta}} W & \sqrt{\tilde{\beta}} \bar{B}^{T} P & 0 \\
* & * & -Z & 0 & 0 \\
* & * & * & Z-2 P & \sqrt{\tilde{\beta}} P B_{\Lambda} \\
* & * & * & * & -\epsilon I
\end{array}\right]<0 .
$$

From $(Z-P) Z^{-1}(Z-P) \geq 0$ we know that $-P Z^{-1} P \leq Z-2 P$. This together with (32) leads to

$$
\left[\begin{array}{ccccc}
\operatorname{sym}(P \bar{A}+U) & P \bar{B}-U+W^{T} & \sqrt{\tilde{\beta}} U & \sqrt{\tilde{\beta}} \bar{A}^{T} P & P B_{\Lambda} \\
* & -\operatorname{sym}(W)+\epsilon C_{\Lambda}^{T} \Lambda^{2} C_{\Lambda} & \sqrt{\tilde{\beta}} W & \sqrt{\tilde{\beta}} \bar{B}^{T} P & 0 \\
* & * & -Z & 0 & 0 \\
* & * & * & -P Z^{-1} P & \sqrt{\tilde{\beta}} P B_{\Lambda} \\
* & * & * & * & -\epsilon I
\end{array}\right]<0 .
$$

Performing a congruence transformation to (33) by $\operatorname{diag}\left\{I, I, I, P^{-1} Z, I\right\}$, we readily obtain (12). Therefore, from Lemma 3.1, we know that the closed-loop system is asymptotically stable, and the proof is completed.

Theorem 3.2 transforms the nonlinear matrix inequalities in Lemma 3.1 into a set of solvable conditions. It is worth noting that when the positive scalar $\epsilon$ is given, the conditions in Theorem 3.2 are LMIs over the decision variables $X>0, Y>0, \bar{Z}>0$, $\bar{U}, \bar{W}$, and $\bar{A}_{c}, \bar{A}_{d}, \bar{B}_{c}, \bar{C}_{c}$, which can be solved via standard numerical software. When these conditions are solvable, a desired output-feedback controller can readily be constructed based on (28). It is worth noting that the synthesis procedure is different from [11] and is much easier to solve than [11]. 

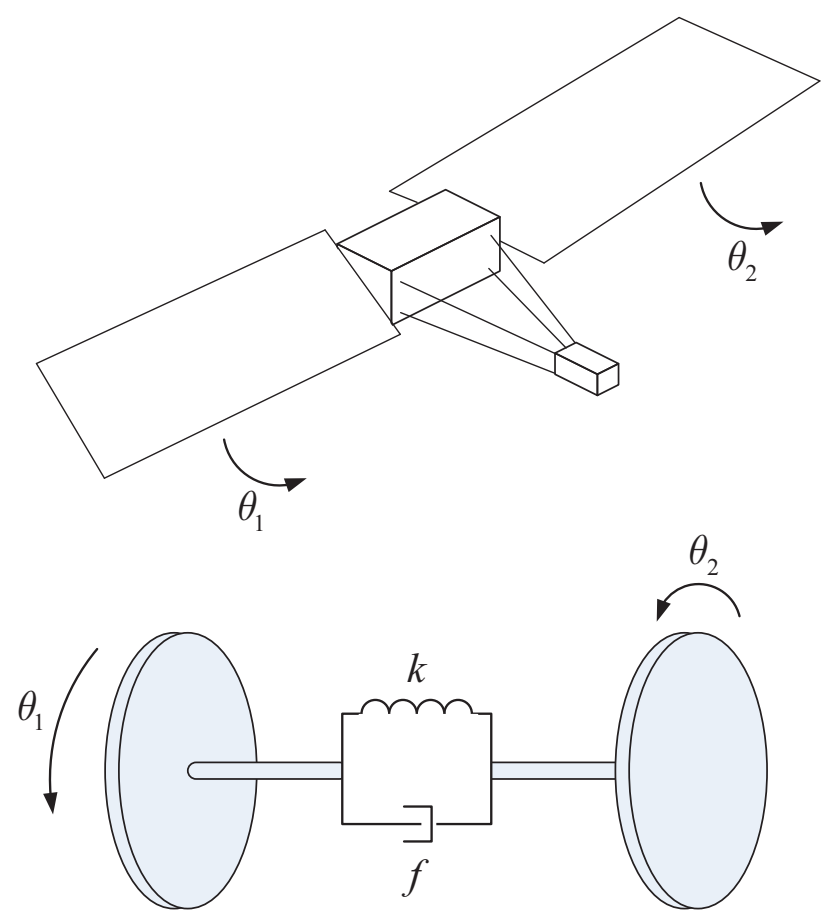

FIG. 3. A satellite system.

4. Illustrative example. In this section, we provide an example to illustrate the output-feedback controller design method proposed in the above section.

Suppose the physical plant in Figure 1 is a satellite system shown in Figure 3 (borrowed from $[2,7]$ ). The satellite system consists of two rigid bodies joined by a flexible link. This link is modeled as a spring with torque constant $k$ and viscous damping $f$. Denoting the yaw angles for the two bodies (the main body and the instrumentation module) by $\theta_{1}$ and $\theta_{2}$, the control torque by $u(t)$, and the moments of inertia of the two bodies by $J_{1}$ and $J_{2}$, the dynamic equations are given by

$$
\begin{aligned}
& J_{1} \ddot{\theta}_{1}(t)+f\left(\dot{\theta}_{1}(t)-\dot{\theta}_{2}(t)\right)+k\left(\left(\theta_{1}(t)-\theta_{2}(t)\right)\right)=u(t), \\
& J_{2} \ddot{\theta}_{2}(t)+f\left(\dot{\theta}_{1}(t)-\dot{\theta}_{2}(t)\right)+k\left(\left(\theta_{1}(t)-\theta_{2}(t)\right)\right)=0 .
\end{aligned}
$$

A state-space representation of the above equation is given by

$$
\begin{aligned}
& J_{1} \ddot{\theta}_{1}(t)+f\left(\dot{\theta}_{1}(t)-\dot{\theta}_{2}(t)\right)+k\left(\left(\theta_{1}(t)-\theta_{2}(t)\right)\right)=u(t), \\
& J_{2} \ddot{\theta}_{2}(t)+f\left(\dot{\theta}_{1}(t)-\dot{\theta}_{2}(t)\right)+k\left(\left(\theta_{1}(t)-\theta_{2}(t)\right)\right)=0 .
\end{aligned}
$$

A state-space representation of the above equation is given by

$$
\operatorname{diag}\left\{1,1, J_{1}, J_{2}\right\}\left[\begin{array}{c}
\dot{\theta}_{1}(t) \\
\dot{\theta}_{2}(t) \\
\ddot{\theta}_{1}(t) \\
\ddot{\theta}_{2}(t)
\end{array}\right]=\left[\begin{array}{cccc}
0 & 0 & 1 & 0 \\
0 & 0 & 0 & 1 \\
-k & k & -f & f \\
k & -k & f & -f
\end{array}\right]\left[\begin{array}{c}
\theta_{1}(t) \\
\theta_{2}(t) \\
\dot{\theta}_{1}(t) \\
\dot{\theta}_{2}(t)
\end{array}\right]+\left[\begin{array}{c}
0 \\
0 \\
u(t) \\
0
\end{array}\right] .
$$

Here we choose $J_{1}=J_{2}=1, k=0.09$, and $f=0.04$ (the values of $k$ and $f$ are chosen within their respective ranges). It is assumed that only $\theta_{2}(t)$ can be measured online. 
Then, the corresponding matrices are given by

$$
A=\left[\begin{array}{cccc}
0 & 0 & 1 & 0 \\
0 & 0 & 0 & 1 \\
-0.09 & 0.09 & -0.04 & 0.04 \\
0.09 & -0.09 & 0.04 & -0.04
\end{array}\right], \quad B=\left[\begin{array}{l}
0 \\
0 \\
1 \\
0
\end{array}\right], \quad C=\left[\begin{array}{llll}
0 & 1 & 0 & 0
\end{array}\right] .
$$

The eigenvalues of $A$ are $-0.04+0.4224 j,-0.0400-0.4224 j, 0,0$; thus, the above system is not stable. Our purpose is to design an output-feedback controller in the form of (10) such that the closed-loop system is asymptotically stable. The following network-related parameters are assumed: the sampling period $h=10$ millisecond (ms); the network-induced delay bound in (2) is given by $\bar{\beta}=15 \mathrm{~ms}$; the maximum number of data packet dropouts $\bar{\delta}=2$; the parameter for the quantizer $f(\cdot)$ is assumed to be $\rho=0.9$; and $u_{0}=2$.

By solving the feasibility problem in Theorem 3.2 for $\epsilon=1$, we obtain the following matrices (for space consideration we do not list all the obtained matrices here):

$$
\begin{aligned}
X & =\left[\begin{array}{cccc}
36.8636 & -6.0262 & -8.4119 & -4.9553 \\
-6.0262 & 38.8203 & 4.4085 & -4.4118 \\
-8.4119 & 30.2706 & 4.4085 & -3.1743 \\
-4.9553 & -4.4118 & -3.1743 & 5.1433
\end{array}\right], \\
Y & =\left[\begin{array}{cccc}
6.0718 & -4.0657 & -1.2830 & -4.4878 \\
-4.0657 & 6.2637 & -2.5465 & -2.4444 \\
-1.2830 & -2.5465 & 49.6595 & -6.5841 \\
-4.5878 & -2.4444 & -6.5841 & 45.3082
\end{array}\right], \\
\bar{A}_{c} & =\left[\begin{array}{cccc}
-0.2677 & -0.0654 & 0.3329 & -0.0297 \\
0.0112 & -0.0800 & -0.0064 & 0.2436 \\
-0.9668 & -0.2173 & -0.3326 & 0.0463 \\
-0.0376 & -0.5688 & 0.3339 & -0.2509
\end{array}\right], \\
\bar{A}_{d} & =\left[\begin{array}{cccc}
0.2548 & 0.0618 & -0.2398 & -0.0577 \\
0.0059 & -0.0483 & -0.1022 & -0.1349 \\
-0.0163 & 0.2324 & 0.3340 & -0.0847 \\
0.0574 & -0.3850 & -0.3528 & 0.2695
\end{array}\right], \\
\bar{B}_{c} & =\left[\begin{array}{c}
-0.0063 \\
-0.9172 \\
-0.0475 \\
-0.0113
\end{array}\right], \quad \bar{C}_{c}=\left[\begin{array}{cc}
-23.0578 \\
-0.8367 \\
-24.2581 \\
-0.3397
\end{array}\right] .
\end{aligned}
$$

Thus, according to (28), the matrices for the output-feedback controller in (10) are given by

$$
\begin{aligned}
A_{c}= & {\left[\begin{array}{cccc}
-0.6155 & -0.0445 & 0.8316 & -0.0967 \\
-0.1861 & -0.2324 & 0.1923 & 0.1093 \\
-1.1054 & 0.0156 & -0.5318 & -0.0530 \\
-0.7230 & -0.9523 & 0.6846 & -0.1081
\end{array}\right], } \\
A_{d}= & {\left[\begin{array}{llll}
0.1252 & -0.8541 & -0.0869 & 0.1006 \\
0.1093 & -0.7373 & -0.0763 & 0.0868 \\
0.0598 & -0.4079 & -0.0417 & 0.0480 \\
0.3203 & -2.1761 & -0.2219 & 0.2555
\end{array}\right], }
\end{aligned}
$$

Copyright $@$ by SIAM. Unauthorized reproduction of this article is prohibited. 


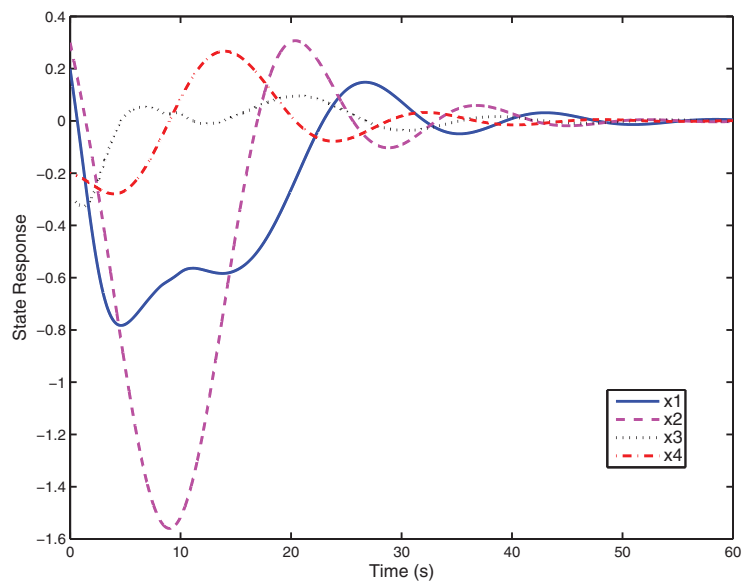

FIG. 4. State responses of closed-loop system.

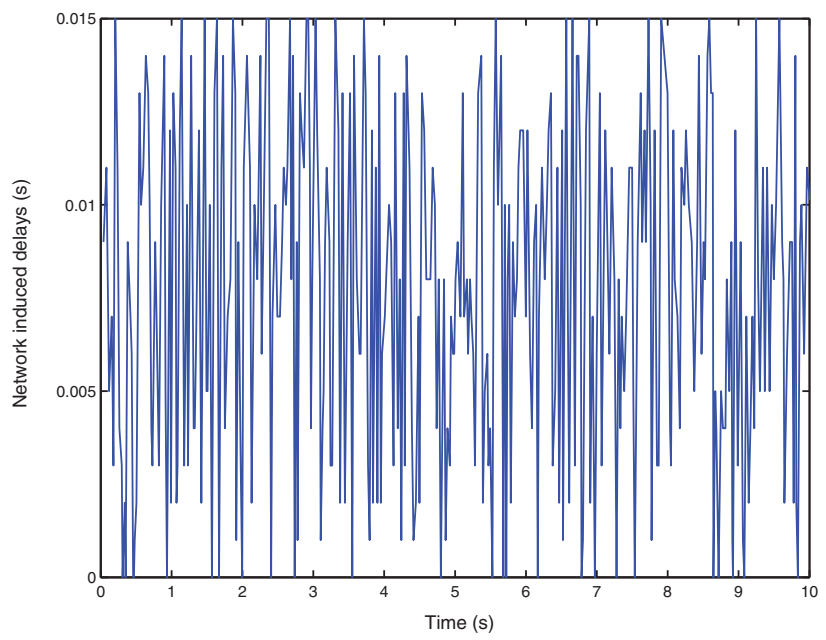

FIG. 5. Network-induced delays.

$$
B_{c}=\left[\begin{array}{l}
0.0220 \\
0.0190 \\
0.0105 \\
0.0562
\end{array}\right], \quad C_{c}=\left[\begin{array}{c}
-23.0578 \\
-0.8367 \\
-24.2581 \\
-0.3397
\end{array}\right]^{T}
$$

Simulations are carried out by connecting the above-obtained output-feedback controller to the physical plant. The initial condition is assumed to be $\left[\begin{array}{ll}0.2 & 0.3\end{array}\right.$ $\left.\begin{array}{ll}-0.3 & -0.2\end{array}\right]^{T}$. The state responses are depicted in Figure 4, from which we can see that all the four state components of the closed-loop system converge to zero. In the simulation, the network-induced delays and the data packet dropouts are generated randomly (uniformly distributed within their ranges) according to the above assumptions and shown in Figures 5 and 6 . The measurement $y(t)$ and the successfully transmitted signal arriving at the $\mathrm{ZOH}$ (denoted as $y_{Z O H}(t)$ ) are shown in Figure 7 where we can see the discontinuous behavior of the transmitted measurements. 


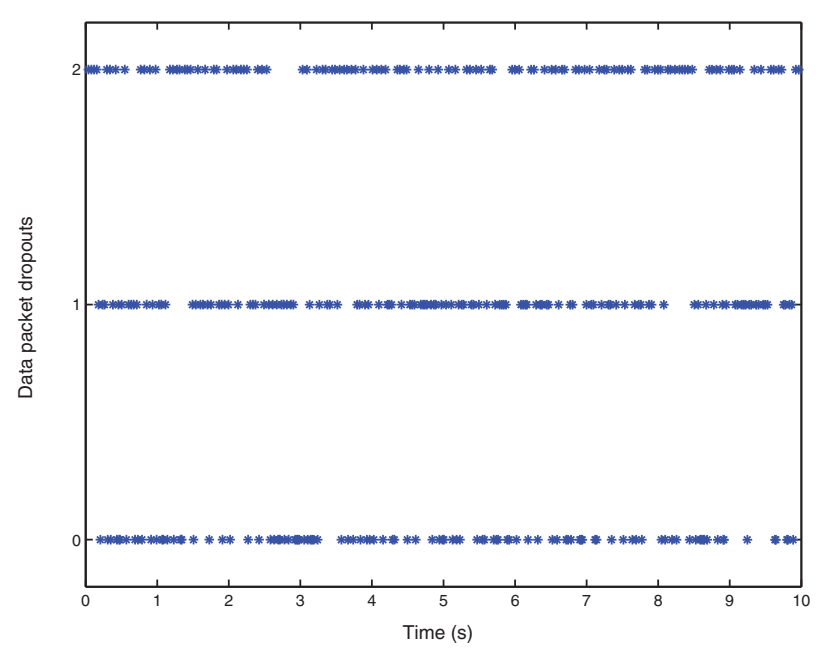

FIG. 6. Data packet dropouts.

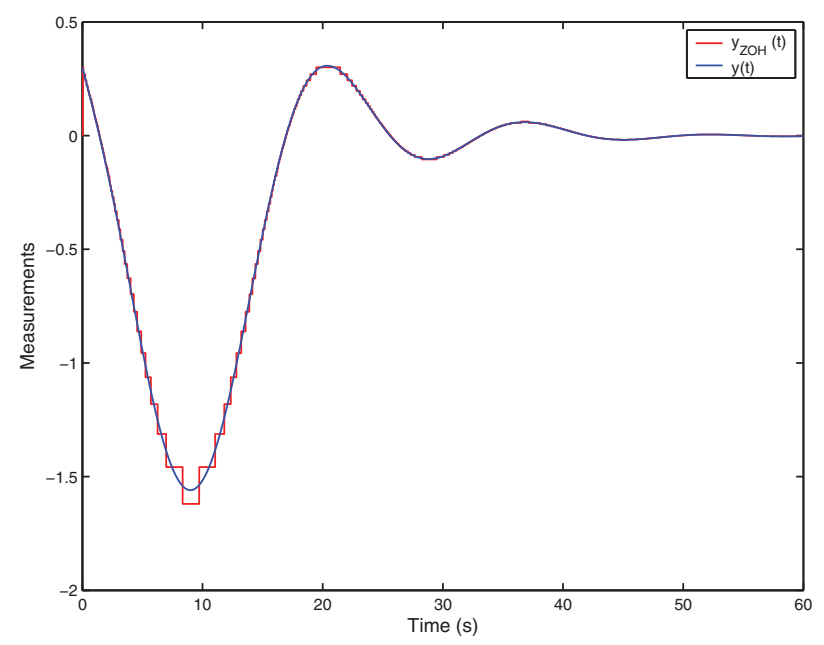

FIG. 7. Measurements and transmitted signals.

5. Conclusions. This paper considers the problem of designing a dynamic linear controller to stabilize an linear time-invariant (LTI) plant. The physical plant and the dynamic controller are in continuous time, and a communication channel exists between the output of the physical plant and the input of the dynamic controller. Attention is focused on the design of dynamic output-feedback controllers, which guarantee the closed-loop networked control systems to be asymptotically stable for all admissible measurement quantizations except time stamps, signal transmission delays, and data packet dropouts, which appear typically in a networked environment. LMI-based conditions have been formulated for the existence of stabilizing outputfeedback controllers. If these conditions are satisfied, a desired controller can be readily constructed. A satellite system is exploited to illustrate the applicability and effectiveness of the controller design methodologies proposed in this paper. 


\section{REFERENCES}

[1] P. Antsaklis and J. Baillieul, Guest editorial. Special issue on networked control systems, IEEE Trans. Automat. Control, 49 (2004), pp. 1421-1423.

[2] R. M. Biernacki, H. Hwang, And S. P. Battacharyya, Robust stability with structured real parameter perturbations, IEEE Trans. Automat. Control, 32 (1987), pp. 495-506.

[3] G. Boukarim, S. Wang, J. Chow, G. Taranto, and N. Martins, A comparison of classical, robust, and decentralized control designs for multiple power system stabilizers, IEEE Trans. Power Syst., 15 (2000), pp. 1287-1292.

[4] N. Elia, Remote stabilization over fading channels, Systems Control Lett., 54 (2005), pp. 237249.

[5] N. Elia AND S. MitTer, Stabilization of linear systems with limited information, IEEE Trans. Automat. Control, 46 (2001), pp. 1384-1400.

[6] M. FU AND L. XIE, The sector bound approach to quantized feedback control, IEEE Trans. Automat. Control, 50 (2005), pp. 1698-1711.

[7] P. Gahinet, A. Nemirovskit, A. J. Laub, and M. Chilali, LMi Control Toolbox User's Guide, The Math. Works, Natick, MA, 1995.

[8] H. Gao And T. Chen, Network-based $H_{\infty}$ output tracking control, IEEE Trans. Automat. Control, 53 (2008), pp. 655-667.

[9] H. Gao, T. Chen, And J. LAM, A new delay system approach to network-based control, Automatica, 44 (2008), pp. 39-52.

[10] H. GaO, J. LAM, AND C. WANG, Robust energy-to-peak filter design for stochastic time-delay systems, Systems Control Lett., 55 (2006), pp. 101-111.

[11] H. GaO, J Lam, C. WANG, AND Y. WANG, Delay-dependent output-feedback stabilisation of discrete-time systems with time-varying state delay, IEE Proc. - Control Theory Appl., 151 (2004), pp. 691-698.

[12] G. Goodwin, H. Haimovich, D. Quevedo, and J. Welsh, A moving horizon approach to networked control system design, IEEE Trans. Automat. Control, 49 (2004), pp. 14271445.

[13] Y. He, M. Wu, J. H. She, And G. P. Liu, Delay-dependent robust stability criteria for uncertain neutral systems with mixed delays, Systems Control Lett., 51 (2004), pp. 57-65.

[14] Y. He, M. Wu, J. H. She, And G. P. LiU, Parameter-dependent Lyapunov functional for stability of time-delay systems with polytopic-type uncertainties, IEEE Trans. Automat. Control, 49 (2004), pp. 828-832.

[15] H. Ishi AND B. Francis, Limited data rate in control systems with networks, Lecture Notes in Control and Inform. Sci. 275, Springer-Verlag, Berlin, Heidelberg, 2002.

[16] F. Lian, J. Moyne, And D. Tilbury, Modelling and optimal controller design of networked control systems with multiple delays, Internat. J. Control, 76 (2003), pp. 591-606.

[17] L. Lu, L. XIE, AND W. CAI, $H_{2}$ controller design for networked control systems, Asian J. Control, 6 (2004), pp. 88-96.

[18] G. NAIR AND R. Evans, Exponential stabilisability of finite-dimensional linear systems with limited data rates, Automatica, 39 (2003), pp. 585-593.

[19] P. Seiler And R. Sengupta, An $H_{\infty}$ approach to networked control, IEEE Trans. Automat. Control, 50 (2005), pp. 356-364.

[20] B. Sinopoli, L. Schenato, M. Franceschetti, K. Poolla, M. Jordan, and S. Sastry, Kalman filtering with intermittent observations, IEEE Trans. Automat. Control, 49 (2004), pp. 1453-1464.

[21] S. TAtikonda And S. Mitter, Control under communication constraints, IEEE Trans. Automat. Control, 49 (2004), pp. 1056-1068.

[22] S. Tatikonda, A. Sahai, AND S. MitTer, Stochastic linear control over a communication channel, IEEE Trans. Automat. Control, 49 (2004), pp. 1549-1561.

[23] Y. Tipsuwan and M. Chow, Control methodologies in networked control systems, Control Engrg. Pract., 11 (2003), pp. 1099-1111.

[24] G. Walsh, H. Ye, AND L. Bushnell, Stability analysis of networked control systems, IEEE Trans. Control Syst. Technol., 10 (2002), pp. 438-446.

[25] Z. WANG, D. W. C. Ho, AND X. LiU, Variance-constrained control for uncertain stochastic systems with missing measurement, IEEE Trans. Syst., Man and Cybernet. Part A, 35 (2005), pp. 746-753.

[26] Z. WANG, F. YAnG, D. W. C. Ho, AND X. LiU, Robust finite-horizon filtering for stochastic systems with missing measurements, IEEE Signal Process. Lett., 12 (2005), pp. 437-440.

[27] L. XIE, Output feedback $H_{\infty}$ control of systems with parameter uncertainty, Internat. J. Control, 63 (1996), pp. 741-750.

Copyright (c) by SIAM. Unauthorized reproduction of this article is prohibited. 
[28] F. YANG, Z. WANG, Y. Hung, AND M. GANI, $H_{\infty}$ control for networked systems with random communication delays, IEEE Trans. Automat. Control, 51 (2006), pp. 511-518.

[29] T. YANG, Networked control system: A brief survey, IEE Proc. - Control Theory Appl., 153 (2006), pp. 403-412.

[30] M. Yu, L. Wang, T. Chu, and F. HaO, Stabilization of networked control systems with data packet dropout and transmission delays: Continuous-time case, Eur. J. Control, 11 (2005), pp. $40-55$.

[31] D. YUE, Q. HAN, AND J. LAM, Network-based robust $H_{\infty}$ control of systems with uncertainty, Automatica, 41 (2005), pp. 999-1007.

[32] L. Zhang, Y. Shi, T. Chen, and B. Huang, A new method for stabilization of networked control systems with random delays, IEEE Trans. Automat. Control, 50 (2005), pp. 11771181.

[33] W. Zhang, M. Branicky, and S. Phillips, Stability of networked control systems, IEEE Control Syst. Mag., 21 (2001), pp. 84-99.

[34] P. Zhivoglyadov and R. Middleton, Networked control design for linear systems, Automatica, 39 (2003), pp. 743-750.

Copyright $@$ by SIAM. Unauthorized reproduction of this article is prohibited. 